\title{
Who is accessing antiretroviral therapy in Malawi? A study in the Southern Region on the occupation category "other"
}

\author{
TB Teferra ${ }^{1}$, M Hochgesang 2 , SD Makombe ${ }^{3}$, K Kamoto ${ }^{3}$, AD Harries ${ }^{3,45}$
}

1 Antiretroviral Supervisor South Region, Zomba Central Hospital, Zomba, Malawi

2 U.S. Centres for Disease Control and Prevention, Global AIDS

Program, Lilongwe, Malawi

3 HIV Unit, Ministry of Health, Lilongwe, Malawi

4 Family Health International, Malawi office, Lilongwe, Malawi

5 London School of Hygiene and Tropical Medicine, London, UK

Correspondence: Professor AD Harries, Family Health International, Malawi Country Office, Arwa House, 3rd Floor, PO Box 30455,

Lilongwe 3, Malawi

Fax: + 2651774307

Email: adharries@malawi.net

\section{Summary}

As part of quarterly national reports on the scale up of antiretroviral therapy (ART), demographic and clinical characteristics are recorded including data on occupation. The largest occupational category is that of "other". As there is no information on the composition of the different occupations of patients placed in this category, a formal study was therefore conducted in 6 representative public sector facilities in the Southeastern Region of Malawi. Between January to June 2006, there were 126 adult patients recorded as "other" in the occupation column. A great variety of different occupations was recorded including no employment $30 \%$, administration jobs $24 \%$, general labourers $11 \%$, builders $10 \%$, tailors $9 \%$ and drivers $7 \%$. A wide range of people with different jobs are accessing ART, and this should help in improving the economy of the patients as well as the country at large.

\section{Introduction}

Sub-Saharan Africa, home to $10 \%$ of the world's population, bears the brunt of the global HIV/AIDS pandemic. Out of $38,600,000$ people infected globally with HIV, the large majority $(24,500,000)$ live in sub-Saharan Africa ${ }^{1}$. Since 2003 , impressive progress has been made in scaling up highly active antiretroviral therapy (ART) for HIV-infected eligible patients in the Africa region, and the number of patients ever started on treatment has increased from 100,000 in 2003 to 1.3 million by the end of $2006^{2}$.

Malawi, a small land-locked country in Southern Africa, has also been making impressive progress with scaling up ART. At the beginning of 2004, an estimated 4,000 patients had ever been started on ART, the number started on free therapy increased to 81,821 patients by December $2006^{3}$. The HIV Unit of Ministry of Health and its partners (Taiwan Medical Mission, Centres for Disease Control, Lighthouse, ART supervisors and MSF-Brussels in Thyolo) collect case finding data on who is accessing ART from all ART sites on a quarterly basis, and this includes data on certain key occupations (Table 1) which is used as a proxy for socioeconomic status and for monitoring access of different groups to ART in Malawi. Almost one third of adults and children are recorded under an occupation category termed "other". There is no national
Table 1 Occupations of patients ever started on antiretroviral therapy in Malawi: cumulative data up to December $31^{t}, 2006$

\begin{tabular}{lll}
\hline Occupation & Number & $\begin{array}{l}\text { Percentage (\% of } \\
\text { known occupations) }\end{array}$ \\
\hline Subsistence farmer & 19,415 & $24.6 \%$ \\
House wife & 15,745 & $19.9 \%$ \\
Small scale business & 10,055 & $12.7 \%$ \\
Student or school child & 3,523 & $4.5 \%$ \\
Teacher & 2,935 & $3.7 \%$ \\
Health care worker & 1,289 & $1.6 \%$ \\
Armed/Police Force & 1,134 & $1.4 \%$ \\
"Other" & 24,854 & $31.6 \%$ \\
Not recorded and unknown & 2,871 & \\
\hline Total & 81,821 & \\
\hline
\end{tabular}

information on the composition of different occupations within this group "other", and we therefore carried out an operational research study in the Southeastern Region of Malawi to document these "other" categories.

\section{Methods}

All health facilities use ART patient master cards and ART registers to routinely collect data on the demographic and clinical characteristics of patients ${ }^{4,5}$. In the ART registers, there is a column termed "Occupation". There are sixteen ART delivering health facilities in the public sector in the South-eastern region of Malawi, and these are supervised clinically by an ART supervisor every three months. These facilities are divided into six strata: namely, central hospitals, district hospitals, mission hospitals, police/military hospitals, health centres and clinics. Of these, six facilities from each strata were selected by simple random sampling to be representative of the facilities delivering ART in the region :- Zomba Central Hospital, Machinga District Hospital, St Martin's Mission Hospital, Zomba Police Hospital, Chipini Health Center and Andiamo Comfort Clinic. All these facilities were visited in early 2007, and occupation information was collected on all adult patients (aged 15 years and above) who were started on ART with an occupation termed "other" between January 1st and June 30th, 2006. Children who were 14 years and below and adult patients who had started on ART sometime previously in another facility and had transferred in to the study facility were excluded from the analysis.

Information on each patient was collected into a structured proforma. Specific data collected for this study did not include personal identifiers. The Malawi National Health Science Research Committee provides approval for the collection and use of routine programmatic data for monitoring and evaluation, and as such no formal submission was made to the committee for ethical approval of this study. The U.S. 
Centres for Disease Control and Prevention also considered this study as programme evaluation, which does not constitute human-subjects research.

\section{Results}

Between January and June 2006, there were a total of 1326 patients (1239 adults and 87 children) who started ART in the six sites. Of these, there were 126 patients (44 female and 82 male, mean age 37 years) started on ART between January to June 2006 with an occupation termed "other". The composition of their occupations is shown in Table 2. Nearly one third of the patients were recorded as unemployed with no work, with the remainder having a variety of different jobs.

\section{Discussion}

This is the first survey in the country of "other" occupations in HIV-infected patients who have been started on ART. As the results show, there is a wide range of different occupations ranging from those who have no regular employment to those engaged in various entrepreneurial or other jobs. When the concept of ART scale up was first discussed at national meetings 4 to 5 years ago, there was concern that the life saving medication would only be for the wealthy and influential sectors of society. With the medication in the public sector being free at the point of delivery, it is reassuring to see after 3 years that ART is in fact being accessed by diverse sectors of society.

Just under $10 \%$ of patients started on ART in the Southeast region had an occupation category of "other", which is significantly less than the $30 \%$ of patients from the national cohort. This is explained by two main factors: a) in the national cohort, children are usually classified as "other", while this particular study focused on adults only: b) a large proportion of "other" patients are registered in the large ART facilities such as Lighthouse, Queen Elizabeth Central Hospital, Thyolo District Hospital and Chiradzulu: these sites, which were not included in the current study, together account for nearly $30 \%$ of patients in the country. In December 2004, during the early days of ART scale up, occupation was recorded from a small number of district and mission hospitals only, and at that time $14 \%$ of patients were classified as "other" (ARV therapy in Malawi - up to December 2004). Patients with "other" were not missed in the six selected sites, and we feel that because the sites were a representative sample of ART facilities in the country, the findings should reflect what is happening nationally. However, the sample size is small and this is a limitation of the study.

We have not seen national reports from other sub-Saharan African countries on occupations of patients starting ART, and we feel that this aspect of the Malawi ART programme
Table 2 Different occupations of patients started on antiretroviral therapy in the Southeastern region of Malawi who had been registered under the category of "Other"

\begin{tabular}{lll}
\hline Occupation & Number & Percentage \\
\hline Unemployed and not at work & 38 & $30.1 \%$ \\
Administration (clerk, secretary) & 30 & $23.8 \%$ \\
General labourer & 14 & $11.1 \%$ \\
Builder & 13 & $10.3 \%$ \\
Tailor & 11 & $8.7 \%$ \\
Driver & 9 & $7.1 \%$ \\
Domestic worker & 6 & $4.9 \%$ \\
Member of Parliament & 1 & $0.8 \%$ \\
Pastor & 1 & $0.8 \%$ \\
Surveyor & 1 & $0.8 \%$ \\
Author & 1 & $0.8 \%$ \\
Forester & 1 & $0.8 \%$ \\
\hline Total & 126 & $100 \%$ \\
\hline
\end{tabular}

is unique. Whether Malawi's ART monitoring and reporting proforma should now be expanded to include some "other" categories will require discussion and debate.

By December 31st 2006, there were almost 60,000 patients alive and on therapy, of whom $96 \%$ were capable of work (source - HIV Unit, Ministry of Health). Without ART many of these patients, who presented with advanced HIV disease or AIDS, would by now have died ${ }^{6}$. It appears therefore that ART is making an important contribution to preserving the fabric of society and improving the economy of the country, although the quantification of this contribution requires a formal and proper social and economic analysis.

\section{Acknowledgement}

Funding for this study was provided from an anonymous donor.

\section{References}

1. UNAIDS. 2006. Report on the global AIDS Epidemic.

2. WHO / UNAIDS / UNICEF. Towards universal access. Scaling up priority HIV/AIDS interventions in the health sector. Progress Report, April 2007

3. Ministry of Health. ART in the public sector in Malawi - results up to 31st December 2006. HIV Unit Report, Lilongwe, Malawi.

4. Libamba E, Makombe S, Harries AD, et al. Scaling up antiretroviral therapy in Africa: learning from tuberculosis control programmes - the case of Malawi. Int J Tuberc Lung Dis 2005; 9: 1062 - 1071

5. Ministry of Health. Treatment of AIDS. Guidelines for the use of antiretroviral therapy in Malawi. Second Edition, April 2006. Lilongwe, Malawi.

6. van Oosterhout JJ, Laufer MK, Graham SM, et al. A communitybased study of the incidence of trimethoprim-sulfamethoxazolepreventable infections in Malawian adults living with HIV. J Acquir Immune Defic Syndr 2005; 39: 626 - 631. 